\title{
Signatures of tunneling and multiphoton ionization in the electron-momentum distributions of atoms by intense few-cycle laser pulses
}

\author{
M. Wickenhauser, ${ }^{1,2}$ X. M. Tong, ${ }^{1,3}$ D. G. Arbó, ${ }^{2}$ J. Burgdörfer, ${ }^{2}$ and C. D. Lin ${ }^{1}$ \\ ${ }^{1}$ J. R. Macdonald Laboratory, Physics Department, Kansas State University, Manhattan, Kansas 66506-2604, USA \\ ${ }^{2}$ Institute for Theoretical Physics, Vienna University of Technology, A-1040 Vienna, Austria \\ ${ }^{3}$ Institute of Materials Science, Graduate School of Pure and Applied Science and Center for Computational Sciences, \\ University of Tsukuba, 1-1-1 Tennodai, Tsukuba, Ibaraki 305-8577, Japan
}

(Received 15 January 2006; revised manuscript received 10 August 2006; published 12 October 2006)

\begin{abstract}
Electron-momentum distributions for above-threshold ionization of argon in a few-cycle, linearly polarized laser pulse are investigated. Spectral features characteristic of multiphoton as well as tunneling ionization coexist over a range of the Keldysh parameter $\gamma$ in the transition regime $\gamma \sim 1$. Surprisingly, the simple strong-field approximation (SFA) is capable of reproducing the key features of the two-dimensional momentum distributions found in the full solution of the time-dependent Schrödinger equation, despite the fact that SFA is known to severely underestimate the total ionization probability.
\end{abstract}

DOI: 10.1103/PhysRevA.74.041402

PACS number(s): $32.80 . \mathrm{Rm}, 32.80 . \mathrm{Fb}, 42.50 . \mathrm{Hz}$

When an atom is placed in an intense laser field, an electron can be ionized through either a multiphoton or a tunneling mechanism. The distinction is based on the so-called Keldysh parameter $\gamma=\sqrt{I_{p} /\left(2 U_{p}\right)}$, where $I_{p}$ is the ionization energy and $U_{p}$ is the ponderomotive potential. In the multiphoton regime, which is characterized by $\gamma \gg 1$, it has been shown that an electron can absorb more photons than necessary in a process called above-threshold ionization (ATI) [1]. The resulting photoelectron spectra show the characteristic ATI peaks separated by the photon energy and shifted by the ponderomotive potential. Later studies have shown fine structure within each ATI peak. These structures, called Freeman resonances [2], have been attributed to transient resonances of ac Stark-shifted Rydberg states. The electron spectra for longer pulses, as well as their angular distributions, are now considered to be well understood in the multiphoton regime; for example, see Ref. [3]. However, recent measurements of the electron spectra for few-cycle pulses in the tunneling ionization regime revealed surprising results [4]. In these experiments, the momentum distributions along the laser polarization direction were measured. According to the tunneling ionization theory one expects smooth spectra, which peak at zero momentum. Instead, resonantlike peaks were found and a double-hump structure centered around $p_{\|}=0$ was observed [4]. The double-hump structure was first attributed to a rescattering process [5]. Subsequently, based on a quasiclassical calculation, it was attributed to the effect of the Coulomb potential on the outgoing electron [6]. Experimentally, these structures were explained by Freeman resonances [7]. In Refs. [8,9] they were considered to be evidences of photon-absorption thresholds in the tunneling regime. Transversal momentum distributions have been studied e.g. in $[6,10]$ where it was shown that the Coulomb interaction leads to a cusplike peak.

In this Rapid Communication we analyze structures in the two-dimensional (2D) momentum distribution and its projection on the laser polarization direction, for different intensities and wavelengths. We present results obtained by solving the time-dependent Schrödinger equation (TDSE) numerically and by using the standard strong-field approximation
(SFA), i.e., without introducing any "corrections" to account for the Coulomb potential. By normalizing the peak of the electron spectra from the SFA to that from the TDSE, we find the surprising result that the two spectra appear to agree quite well. In the momentum spectra, we find "resonancelike" structures that can be attributed to multiphoton ionizations on top of a smooth "background" due to tunneling ionization. However, sharp structures can also come from Freeman resonances for longer pulses and from incomplete constructive interferences for few-cycle pulses [12,13]. We illustrate the relative importance of tunneling vs multiphoton ionization features as the Keldysh parameter is varied.

Atomic units $\left(m_{e}=\hbar=e=1\right)$ are used throughout the paper unless otherwise indicated. The TDSE is solved in the singleactive-electron approximation with the method of $[14,15]$. The effective potential was calculated as described in [16]. We simulate a $10 \mathrm{fs}$ (full width at half maximum) laser pulse with a wavelength for 400 and $800 \mathrm{~nm}$ and a peak intensity in the range $(1.7-3.9) \times 10^{14} \mathrm{~W} / \mathrm{cm}^{2}$. These laser parameters lie in the transition regime from multiphoton to tunneling ionization, with a Keldysh parameter ranging between 1.76 and 0.85 . [17]

In the SFA model the ionization amplitude is given by

$$
b(\mathbf{p})=i \int_{-\infty}^{\infty} d t e^{i \phi_{V}(\mathbf{p}, t)}\langle\mathbf{p}-\mathbf{A}(t)|\mathbf{F}(t) z| g\rangle_{t},
$$

where $\mathbf{A}(t)$ is the vector potential of the laser field. The polarization axis is along the $z$ direction. The Volkov phase in Eq. (1) can be written as $\phi_{V}(\mathbf{p}, t)=-\int_{t}^{\infty} d t^{\prime}\left[\mathbf{p}-\mathbf{A}\left(t^{\prime}\right)\right]^{2} / 2$. We have calculated the dipole moment in Eq. (1) using the energy-scaled hydrogenic $3 p$ wave function. The electric field of the laser pulse can be written in the form $\mathbf{F}(t)=F_{0} \hat{\boldsymbol{\epsilon}} a(t) \cos (\omega t+\varphi)$, with $\hat{\boldsymbol{\epsilon}}$ the polarization vector, $\omega$ the carrier frequency, and $\varphi$ the carrier-envelope phase. The envelope function $a(t)$ is chosen to be $\sin ^{2}\left(\frac{\pi(t+\tau / 2)}{\tau}\right)$ for $-\frac{\tau}{2}<t<\frac{\tau}{2}$ and zero elsewhere. In the following calculations we set the carrier envelope phase to zero. 


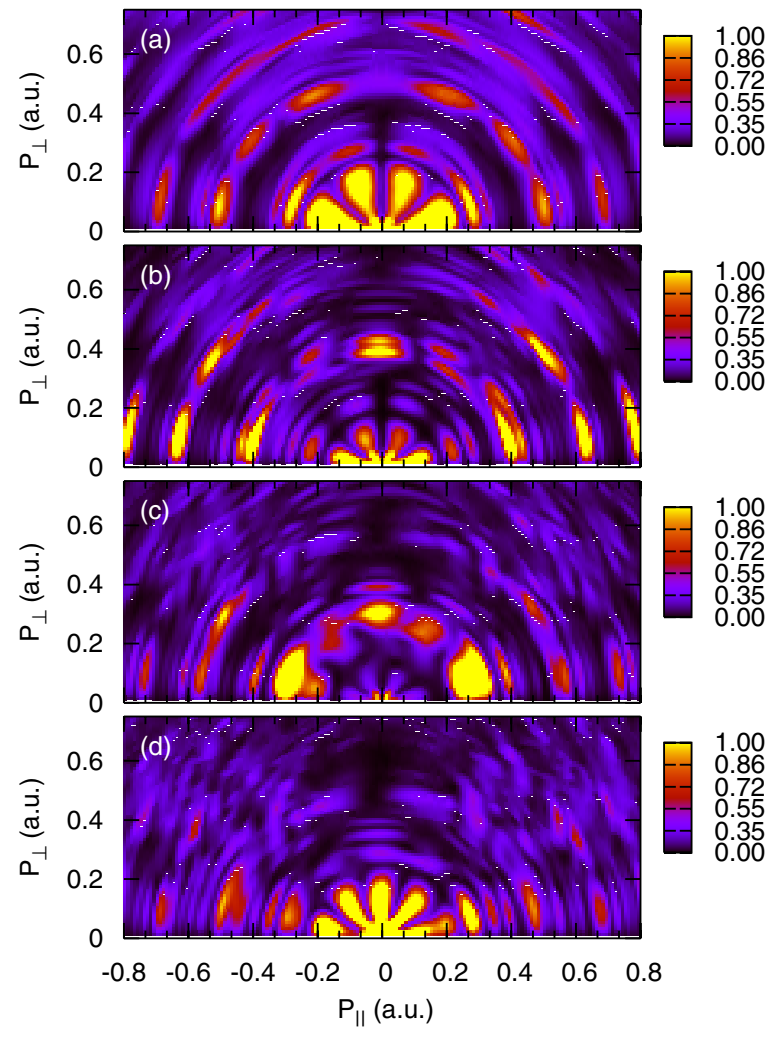

FIG. 1. (Color online) Photoelectron momentum distributions for argon calculated by solving the TDSE for four intensities 1.7, $2.4,3.2$, and $3.9 \times 10^{14} \mathrm{~W} / \mathrm{cm}^{2}$, respectively, for a wavelength of $400 \mathrm{~nm}$.

In Fig. 1 we present the angle-resolved momentum distribution for four different intensities 1.7, 2.4, 3.2, and $3.9 \times 10^{14} \mathrm{~W} / \mathrm{cm}^{2}$, respectively, obtained with the TDSE. The carrier wavelength is $400 \mathrm{~nm}$, and the $\gamma$ parameters for the four intensities are 1.76, 1.48, 1.27, and 1.13. The horizontal axis in the plot is the momentum parallel to the laser field polarization and the vertical axis is the perpendicular momentum defined by $p_{\perp}=\sqrt{p_{x}^{2}+p_{y}^{2}}$. ATI peaks appear as circles of increased ionization probability in the momentum distribution. It can be seen that the ionization probability is not constant on a given circle, but has maxima and minima. This structure reflects the parity and the dominant angular momentum of the emitted electrons at that particular energy $[3,18]$.

For the intensity of $1.7 \times 10^{14} \mathrm{~W} / \mathrm{cm}^{2}$ [Fig. 1(a)] we see rings of constant energy which are easily identified with the ATI peaks in the energy spectra, each with alternating parity (seen from the node or antinode at $p_{\|}=0$ ). Within each ATI peak, we note that there are subpeaks. These subpeaks are caused by the shifting ponderomotive potential during the ionization process in the short pulse, which causes ionization at different times in the pulse not be add up fully coherently $[12,13]$. As seen from Fig. 1(a), they can be distinguished from the neighboring ATI peaks by their different parities. Furthermore, within the first ATI peaks, at small momenta, we note pronounced structures that fan out radially. These fanlike structures have been found to be the major features in the experimental 2D momentum spectra $[10,11]$. For the
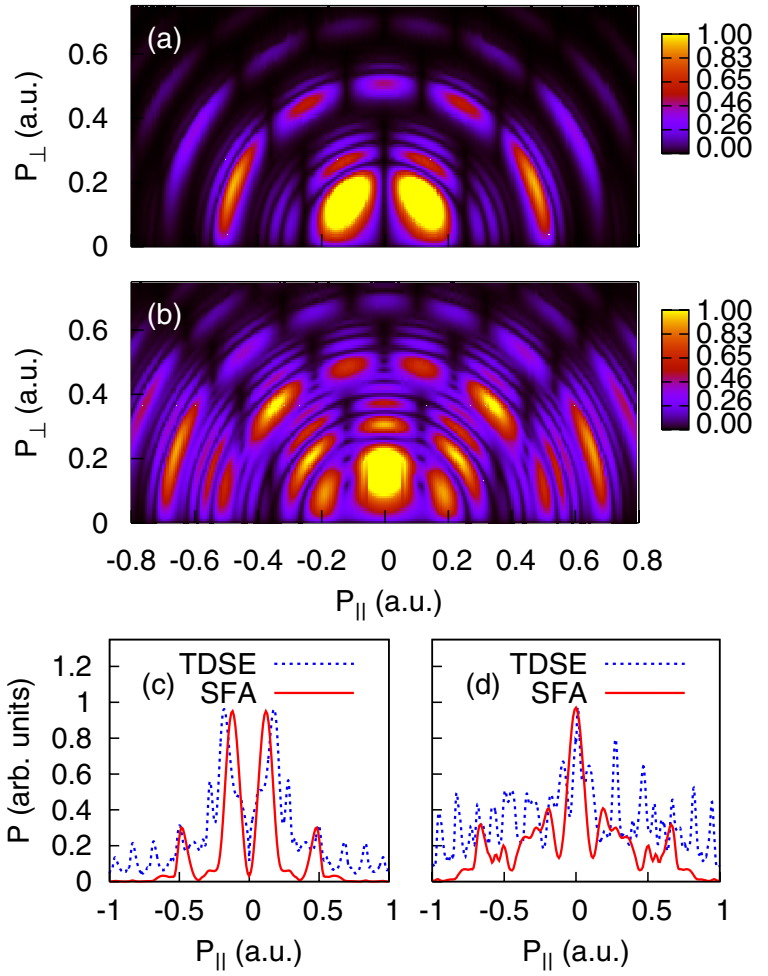

FIG. 2. (Color online) (a) and (b) 2D electron momentum distributions for the intensities 1.7 and $3.9 \times 10^{14} \mathrm{~W} / \mathrm{cm}^{2}$, respectively, for a wavelength of $400 \mathrm{~nm}$ obtained with the SFA model. (c) and (d) Comparison of parallel momentum distributions between the SFA model and the TDSE result, for laser parameters of (a) and (b), respectively.

present case, they are dominated by the $l=3$ angular momentum of the electron.

Understanding the parity of the ATI peaks is straightforward. For the intensity $1.7 \times 10^{14} \mathrm{~W} / \mathrm{cm}^{2}$, six photons are sufficient to ionize the initial $3 p$ state which is of add parity. However, at the intensity $2.4 \times 10^{14} \mathrm{~W} / \mathrm{cm}^{2}$ [Fig. 1(b)] an additional photon is needed for ionization. The "original" odd-parity first ATI peak becomes much weaker, and the next even-parity ATI peak becomes more dominant. That the former is still visible is attributed to the finite width of the ATI peak caused by the short pulse. For the intensity $3.2 \times 10^{14} \mathrm{~W} / \mathrm{cm}^{2}$ the original first ATI peak is hardly visible any more and finally for $I=3.9 \times 10^{14} \mathrm{~W} / \mathrm{cm}^{2}$ the original second ATI peak, with the dominant angular momentum $l=4$ and even parity, has taken over the first position. This process is called channel switching.

To address the question of the influence of the Coulomb force on the momentum distribution, we compare our TDSE results with the SFA model, which does not include the effect of the Coulomb potential. In Figs. 2(a) and 2(b) we show the angle-resolved momentum distributions obtained with the SFA model for two intensities (1.7 and 3.9) $\times 10^{14} \mathrm{~W} / \mathrm{cm}^{2}$, respectively, for a wavelength of $400 \mathrm{~nm}$. At low intensity, we observe a surprisingly general agreement between Figs. 2(a) and 1(a). All ATI peaks with their corresponding substructures in the energy domain and their parities can be understood within the SFA. Major difference occurs in the 
low-energy part of the spectra. In the TDSE result the nodal lines in the momentum distribution of an ATI peak always fan out radially, while in the SFA model they are vertical. A Coulomb-corrected SFA model [9] is able to recover the radial nodal structure. This leads to a sharper structure in the momentum projection in the SFA result as compared to the TDSE.

The behavior of the 2D momentum spectra from SFA can be easily understood. For a pulse of constant field strength $F_{0}$ acting from $0<t<\tau$, the momentum-dependent ionization amplitude $b(\mathbf{p})$ is proportional to [17]

$$
\sum_{n=0}^{\infty} f_{n}\left(p_{\|}\right) \int_{0}^{\tau} d t e^{i\left(\mathbf{p}^{2} / 2-n \omega+I_{p}+U_{p}\right) t}\langle\mathbf{p}-\mathbf{A}(t)|z| g\rangle
$$

The function $f_{n}\left(p_{\|}\right)$is given by

$$
f_{n}\left(p_{\|}\right)=\sum_{m+2 j=-n \pm 1} i^{m} J_{m}\left(-\frac{p_{\|} F_{0}}{\omega^{2}}\right) J_{j}\left(\frac{I}{8 \omega^{3}}\right),
$$

where the sum runs over all $-\infty<m, j<\infty . J_{m}$ is a Bessel function of order $m$. Each term in the sum in Eq. (2) corresponds to a single ATI peak originating from the absorption of $n$ photons, which is located at the energy $\mathbf{p}^{2} / 2=n \omega-I_{p}-U_{p}$. The integral in Eq. (2) alone would result in a ringlike momentum distribution without nodes. The nodal structure within the ATI peaks is caused by the prefactor $f_{n}\left(p_{\|}\right)$. Since $f_{n}\left(p_{\|}\right)$depends only on $p_{\|}$and not on $p_{\perp}$ all nodal lines in the SFA calculation are vertical. While the difference between radial and vertical nodal lines is clearly seen in the ATI peak near threshold, it becomes smaller for higher ATI peaks.

For the higher intensity, the difference between Figs. 1(d) and 2(b) appears to be larger. A closer look finds that the difference is easily understood. In addition to the general difference in the low-energy part, we note that in TDSE the electrons tend to be located more at small $p_{\perp}$, reflecting the greater importance of tunneling ionization.

The projection on the momentum parallel to the polarization direction is presented in Figs. 2(c) and 2(d) for the SFA model as well as from solving the TDSE. The result from the SFA calculation is normalized to the same maximum peak as the TDSE result. We note that the total ionization probability of a model based on SFA is too small and can be improved by multiplying the Volkov state with a correction factor which accounts for tunneling [19]. However, we do not include this factor since we intend to compare our TDSE calculation with a model that does not include any effect of the Coulomb potential. In Fig. 2(c), we clearly see a dip in both calculations, while a peak is visible in Fig. 2(d) in the $p_{\|}$ distribution. The 2D momentum distribution clarifies the behavior of the momentum projection in Figs. 2(c) and 2(d). In Fig. 1(a), the first ATI peak has the highest amplitude and has odd parity. This symmetry leads to a dip in the momentum projection with a maximum on either side. On the contrary, for the intensity $3.9 \times 10^{14} \mathrm{~W} / \mathrm{cm}^{2}$, the momentum projection has a clear peak at $p_{\|}=0$, reflecting the even-parity nature of the first ATI peak, since one more photon is needed for ionization.
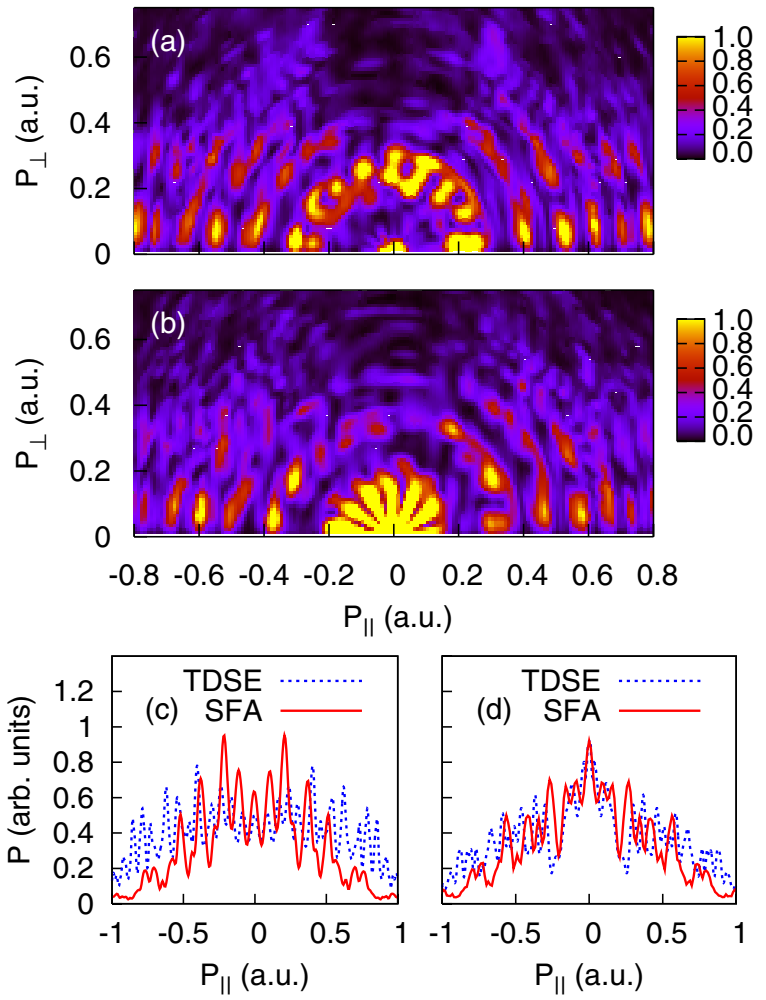

FIG. 3. (Color online) (a) and (b) 2D electron momentum distributions for the intensities $(1.65$ and 1.8$) \times 10^{14} \mathrm{~W} / \mathrm{cm}^{2}$, respectively, for a wavelength of $800 \mathrm{~nm}$ obtained from solving the TDSE. (c) and (d) Comparison of parallel momentum distributions between the TDSE result and the SFA model, for laser parameters of (a) and (b), respectively. The TDSE result is averaged over the carrier-envelope phases 0 and $\pi$.

This allows us to conclude that for these laser parameters the dip in the momentum projection is not caused by the effect of the Coulomb force. In comparing Fig. 2(c) to Fig. 2(d), we note that the latter has a larger smooth background. We can attribute this background to tunneling ionization which increases rapidly with higher laser intensity, or with smaller $\gamma$.

In Figs. 3(a) and 3(b) we present the angle-resolved spectra for a wavelength of $800 \mathrm{~nm}$ and two slightly different intensities of $(1.65$ and 1.8$) \times 10^{14} \mathrm{~W} / \mathrm{cm}^{2}$, corresponding to $\gamma=0.89$ and 0.85 , respectively. First we note that the background continuum is much larger, as compared to the $400 \mathrm{~nm}$ case. This is consistent with the smaller Keldysh parameter which means that tunneling ionization becomes more important. Even in this case the ATI peaks are visible, but the different peaks become now hard to distinguish. We observe both a dip and a peak in Figs. 3(c) and 3(d) for even parity of the first ATI peak. Thus the projection on the parallel momentum is no longer dominated by the parity of the first ATI peak. Moreover, the dip in the projected spectra changes to a central peak with a less than $10 \%$ increase in intensity. For Fig. 3(d), the first ATI peak is close to threshold; for Fig. 3(c) it is about a photon energy away. This shows that in the tunneling regime the position of the first ATI is more relevant in determining the structure in the parallel-momentum projection than the parity of the lowenergy electrons in agreement with [8]. 
How to use the present theoretical results to explain existing experimental data? To begin with, most of the experimental data were taken with longer pulses, about 25-50 fs, and the finite size of the focal volume must be considered in a comparison. For $400 \mathrm{~nm}$, general agreement between our calculation and experimental data [11] has been found, in both the projected momentum distribution and the main features in the 2D spectra. Since each ATI peak is $3.1 \mathrm{eV}$ apart, it appears that the volume effect does not wash out the sharp features. For $800 \mathrm{~nm}$, each ATI peak is separated only by $1.55 \mathrm{eV}$, and the sharp structures predicted in the theory are not expected to be observed after the volume integration. As shown in Fig. 3, a change in intensity of less than $10 \%$ causes a change in the momentum distribution from a peak to a dip. The position of a Freeman resonance, on the other hand, is rather insensitive to the laser intensity and thus can survive the volume effect, which supports the explanation [7] that the structures observed in $[4,7]$ are likely coming from Freeman resonances. Whether the projected momentum spectra have a peak or a dip at $p_{\|}=0$ depends on whether a dominant resonance peak is close to the threshold or not.

In conclusion, we have investigated structures in the electron-momentum spectra for above threshold ionization of argon in a few-cycle laser pulse. We have solved the TDSE and a model based on the SFA for laser parameters in the transition regime from multiphoton to tunneling ionization. In general, we find good agreement between the two approaches. We conclude that outside the near-threshold region the asymptotic Coulomb interaction plays only a minor role in determining the structural features of the ATI momentum spectra and their projection on the parallel momentum. Because of the absence of the smooth background in the multiphoton regime the parity and the dominant angular momentum of the first ATI peak determines the shape of the momentum projection. In the multiphoton regime a dip in the momentum projection is caused by an odd parity of the first ATI peak, relevant for recent experiments [11]. In the tunneling regime our full numerical solution of the TDSE supports the result from [8] that the position of the first ATI peak determines whether a peak or a dip is seen in the momentum projection.

This work was supported in part by Chemical Sciences, Geosciences and Biosciences Division, Office of Basic Energy Sciences, Office of Science, U.S. Department of Energy. M.W. received financial support from the Austrian Academy of Sciences. D.A. and J.B. acknowledge support by the SFBADLIS of the FWF (Austria). The authors greatly acknowledge the help of Dr. Toru Morishita and Dr. Zhangjin Chen.
[1] P. Agostini, F. Fabre, G. Mainfray, G. Petite, and N. K. Rahman, Phys. Rev. Lett. 42, 1127 (1979).

[2] R. R. Freeman, P. H. Bucksbaum, H. Milchberg, S. Darack, D. Schumacher, and M. E. Geusic, Phys. Rev. Lett. 59, 1092 (1987).

[3] R. Wiehle, B. Witzel, H. Helm, and E. Cormier, Phys. Rev. A 67, 063405 (2003).

[4] R. Moshammer et al., Phys. Rev. Lett. 91, 113002 (2003).

[5] J. Chen and C. H. Nam, Phys. Rev. A 66, 053415 (2002).

[6] K. I. Dimitriou, D. G. Arbó, S. Yoshida, E. Persson, and J. Burgdörfer, Phys. Rev. A 70, 061401(R) (2004).

[7] A. Rudenko, K. Zrost, C. D. Schröter, V. L. B. de Jesus, B. Feuerstein, R. Moshammer, and J. Ullrich, J. Phys. B 37, L407 (2004).

[8] F. H. Faisal and G. Schlegel, J. Phys. B 38, L223 (2005).

[9] F. H. Faisal and G. Schlegel, J. Mod. Opt. 53, 207 (2006).
[10] A. Rudenko et al., J. Phys. B 38, L223 (2005).

[11] C. M. Maharjan, A. S. Alnaser, I. Litvinyuk, P. Ranitovic, and C. L. Cocke, J. Phys. B 39, 1955 (2006).

[12] M. Wickenhauser, X. M. Tong, and C. D. Lin, Phys. Rev. A 73, 011401(R) (2006).

[13] J. N. Bardsley, A. Szöke, and M. J. Comella, J. Phys. B 21, 3899 (1988).

[14] X. M. Tong and Shih-I Chu, Chem. Phys. 217, 119 (1997).

[15] X. M. Tong and Shih-I Chu, Phys. Rev. A 61, 031401(R) (2000).

[16] X. M. Tong and C. D. Lin, J. Phys. B 38, 2593 (2005).

[17] M. Lewenstein, P. Balcou, M. Y. Ivanov, A. L'Huillier, and P. B. Corkum, Phys. Rev. A 49, 2117 (1994).

[18] D. G. Arbó, S. Yoshida, E. Persson, K. I. Dimitriou, and J. Burgdörfer, Phys. Rev. Lett. 96, 143003 (2006).

[19] V. P. Krainov and B. Shokri, JETP 80, 657 (1999). 